ISSN 2447-9071

doi $10.36414 /$ rbmc.v5i12.1
Contato para correspondência: Rogério José de Almeida

E-mail:

rogeriopucgo@gmail.com

Conflito de interesse: Não

Financiamento: Recursos próprios

Recebido: 31/05/2019

Aprovado: 07/08/2019

\section{Relação entre 0 ambiente laboral e problemas osteomusculares: um estudo com profissionais de enfermagem em um hospital escola}

\section{Relationship between the labor environment and osteomuscular problems: a study with nursing professionals in a school hospital}

Natália Costa Resende Cunha', Vanessa Cotian Oliveira², Mário Silva de Araújo Filho³, Rogério José de Almeida ${ }^{4}$

\begin{abstract}
'Médica graduada em Medicina pela PUC Goiás, '2Enfermeira Mestre em Ciências Ambientais e Saúde pela PUC Goiás, professora do curso de enfermagem da UniCerrado, ${ }^{3}$ Enfermeiro Mestre em Ciências Ambientais e Saúde pela PUC Goiás, professor do curso de enfermagem da PUC Goiás, ${ }^{4}$ Cientista Social, Doutor em Sociologia, Pós-Doutorando em Ciências da Saúde, Professor do curso de Medicina e do Programa de Pós-Graduação em Ciências Ambientais e Saúde da PUC Goiás, Professor da Faculdade da Polícia Militar.
\end{abstract}

\section{Resumo}

Este estudo teve por objetivo analisar a relação entre ambiente organizacional com os problemas osteomusculares em profissionais de enfermagem em um hospital escola. Trata-se de um estudo transversal analítico com abordagem quantitativa. Do total de 240 trabalhadores deenfermagem, 70 foram excluídos após aplicar os critérios de inclusão e exclusão. A amostra final foi constituída de 119 profissionais de enfermagem ao utilizar um nível de confiança de 95\% e margem de erro de 5\%. Os profissionais responderam questionários sociodemográficos, deinformações laboraiseo Questionário Nórdico de Sintomas Osteomusculares (QSNO). Dos 119 profissionais, 97,5\% eram do sexo feminino, a faixa etária predominante foi de 20 a 35 anos e 63\% relataram ter filho. Um total de 103 pessoas relataram algum sintoma osteomuscular, sendo a região lombar a mais acometida nos últimos 12 meses (60,5\%) e nos últimos 7 dias (43,7\%). A análise dos dados mostrou que aqueles que relataram algum sintoma nos últimos 12 meses também consideravam que o seu ambiente de trabalho trazia algum risco à saúde $(p=0,02)$ e que as pessoas que possuíam filhos se afastaram mais do trabalho do que aquelas que não possuíam filhos ( $p=0,03$ ). Além disso, aqueles que relataram que "costumavam se cansar" apresentaram algum sintoma osteomuscular nos últimos 12 meses $(p<0,01)$ enos últimos sete dias ( $p=0,02$ ). Analisando os dados este estudo mostrou que a percepção de risco à saúde no ambiente de trabalho e sentimento de cansaço ou fadiga foram os fatores implicados em mais sintomas, enquanto que a satisfação no trabalho esteve relacionada a menos queixas álgicas.

Palavras-Chave: Sintomas Osteomusculares, Profissionais de Enfermagem, Ambiente de Trabalho.

\begin{abstract}
This studyaimed to analyze the relationship betweenorganizational environmentandmusculoskeletal problems in nursing professionals in a school hospital. This is an analytical cross-sectional study with a quantitative approach. Of the total of 240 nursing workers, 70 were excluded after applying theinclusion and exclusion criteria. The final sample consisted of 119 nursing professionals using a 95\% confidence level and a 5\% margin of error. The professionals answered sociodemographic questionnaires, labor information questionnaires and the Nordic Osteomuscular Symptoms Questionnaire (QSNO). Of the 119 professionals, $97.5 \%$ werefemale, the predominant age group was 20 to 35 years and $63 \%$ reported having a child. A total of 103 people reported some musculoskeletal symptoms, the lumbar region being the most affected in the last 12 months (60.5\%) and in the last 7 days (43.7\%). Data analysis
\end{abstract}


showed that those who reported some symptom in the last 12 months also considered that their work environment posed some health risk $(p=0.02)$ and that people who had children moved away from work more than those who did not have children $(p=0.03)$. In addition, those who reported that they "used to get tired" had some musculoskeletal symptoms in the last 12 months $(p<0.01)$ and in the last seven days $(p=0.02)$. Analyzing the data, this study showed that the perception of health risk in the work environment and feeling of fatigue or fatigue were the factors implicated in more symptoms, while satisfaction at work was related to fewer pain complaints.

Keywords: Musculoskeletal symptoms, Nursing professionals, Workplace.

\section{Introdução}

Em um mundo onde produzir cada vez mais é a regra, as consequências adversas físicas e emocionais das atividades laborais excessivas também passam a ganhar mais notoriedade. Dentre estas consequências estão os distúrbios osteomusculares relacionados ao trabalho (DORT) também chamados de lesões por esforço repetitivo (LER), caracterizados como lesões causadas ou exacerbadas por várias formas de exposição dentro do ambiente de trabalho. Podem afetar músculos, tendões, sinóvias, nervos, fáscias e ligamentos, isolados ou combinados, com ou sem a degeneração de tecidos e podem se manifestar clinicamente com dor, parestesia, sensação de peso e fadiga'.

O Instituto Brasileiro de Geografia e Estatística (IBGE) estimou em 2013 que mais de 3 milhões de trabalhadores referiram ter sido acometidos por LER/DORT e que há décadas são as doenças ocupacionais mais frequentes nas estatísticas da Previdência Social ${ }^{2}$. Além disso, uma pesquisa do Instituto Nacional do Seguro Social (INSS) entre os anos de 2000 e 2011 identificou que a doença, motivada por fatores de riscos ergonômicos, representou $20,76 \%$ de todos os afastamentos do trabalho. Além do aumento de trabalhadores afetados por estes distúrbios, as LER/DORT afetam também o sistema previdenciário brasileiro ${ }^{3}$.

Dentre os perfis de trabalhadores que sofrem com os efeitos ergonômicos laborais, os enfermeiros merecem destaque, pois estão sujeitos a jornadas longas, muitas vezes fora do ritmo circadiano, prolongada exposição a fatores de risco (físico, biológico, químico e psicossocial), atividades com movimentos repetitivos utilizando-se dos mesmos grupos musculares. Além disso, a alta carga de estresse emocional relacionada à imprevisibilidade das ações e estado de vigília constante são fatores que aumentam a prevalência dos distúrbios osteomusculares nestes profissionais ${ }^{4}$.

Diversas variáveis influenciam a prevalência e intensidade dos sintomas musculoesqueléticos nestes profissionais da saúde. Sexo, idade, presença prévia de alguma desordem, conhecimento sobre medidas a serem feitas para evitar tais sintomas e local de trabalho são algumas delas. Um estudo de coorte na Tailândia, por exemplo, com quase 18 mil enfermeiros, mostrou que os distúrbios musculoesqueléticos nos enfermeiros aumentaram significativamente com a idade, índice de massa corporal e duração do trabalho5. Já um estudo transversal em um hospital no Irã mostrou que apesar de $50,7 \%$ dos enfermeiros participantes terem consciência sobre os princípios de cuidados com dores lombares, $71,8 \%$ apresentavam pelo menos um tipo de dor nas costas, o que mostra que o conhecimento nem sempre é um fator protetor nesse contexto ${ }^{6}$.

Contudo, em se tratando de uma desordem relacionada ao trabalho, espera-se que determinadas funções e locações dos enfermeiros em diferentes setores dentro de um hospital influenciem sobremaneira na incidência de LER/DORT. A ideia é que setores com pacientes mais imobilizados, mais fatores de risco e condições estressoras estejam mais relacionados com mais distúrbios osteomusculares. Uma pesquisa com 7 mil enfermeiros identificou que a prevalência de DORT foi maior no Departamento de Emergência enquanto a prevalência foi menor no Departamento de Clínica Médica ${ }^{7}$. Já em outro estudo, as enfermeiras que trabalhavam em unidades de terapia intensiva foram mais susceptíveis às LER/DORT em comparação com seus colegas de outros setores e isso se deveu ao fato de os pacientes serem totalmente dependentes e imóveis ${ }^{8}$.

Nesse sentido, em se tratando de um problema de saúde pública, as doenças osteomusculares em enfermeiros devem ser exaustivamente estudadas, à medida que quanto mais se sabe sobre os fatores que influenciam, mais é possível criar medidas para evitá-los, melhorando a qualidade da assistência prestada, a qualidade de vida dos profissionais de saúde e diminuindo gastos onerosos com afastamentos previdenciários. E, como visto, ambientes de trabalho diversos dentro de um hospital poder ter vínculo estreito com a incidência destas doenças osteomusculares. 
Sendo assim, este trabalho tem por objetivo analisar a relação entre ambiente organizacional com os problemas osteomusculares em profissionais de enfermagem em um hospital escola.

\section{Métodos}

Trata-se de um estudo transversal analítico com abordagem quantitativa. $O$ estudo transversal é um método de pesquisa que investiga a exposição a fatores de risco ou proteção, bem como a associação desses a indivíduos não expostos a partir de observações pontuais do tempo.

Os participantes da pesquisa foram enfermeiros $(n=25)$ e técnicos de enfermagem $(n=94)$ dos diversos setores da unidade hospitalar. A coleta de dados foi realizada entre os meses de junho a agosto de 2017 e ocorreu durante todos os turnos.

Os critérios de inclusão foram profissionais de enfermagem, ambos os sexos, não possuir doença osteomuscular anterior à admissão no hospital e não possuir doença osteomuscular autoimune (ex.: artrite reumatoide, lúpus). Os critérios de exclusão foram não responder as questões dos questionários e ter faltado ao serviço no dia da aplicação do questionário.

A amostra foi definida após o levantamento do número de trabalhadores em enfermagem de todo o hospital, totalizando 240 indivíduos. Ao aplicar os critérios de inclusão e exclusão restaram 170. Com esta população utilizou-se um nível de confiança de $95 \%$ e margem de erro de $5 \%$. Assim sendo, amostra final foi constituída por 119 profissionais de enfermagem.

Para a coleta de dados foram utilizados: 1) um questionário sociodemográfico ocupacional autoaplicável constituído por itens com informações sociodemográficas (nome, sexo, idade, peso, altura, sedentarismo) e informações laborais (função, turno, carga horária semanal, tempo na função e setor, número de vínculos empregatícios); 2) Questionário Nórdico de Sintomas Osteomusculares (QNSO) que foi desenvolvido na Finlândia, com a proposta de padronizar a mensuração de relato de sintomas osteomusculares e, assim, facilitar a comparação dos resultados entre os diversos estudos sobre o assunto. Esse instrumento já foi validado no Brasil $^{9} \mathrm{e}$ adaptado para cultura brasileira ${ }^{10}$. Tem por objetivo avaliar a ocorrência de sintomas nas diversas regiões anatômicas do corpo nas quais são mais comuns sua ocorrência.

Éum instrumento que avalia, por meio de escolhas múltiplas ou binárias, a ocorrência de sintomas nas diversas regiões anatômicas nas quais são mais comuns sua ocorrência. $O$ entrevistado deve relatar a ocorrência dos sintomas considerando os 12 meses e os sete dias anteriores à entrevista, assim como relatar a ocorrência de afastamento das atividades diárias no último ano, bem como o nível de dor quando da existência de sintomas.

Os escores são obtidos para cada um dos fatores da escala
(12 meses, últimos sete dias e afastamento), variando de nove (nenhum sintoma) a 24. Quanto maior o escore, maior também é a ocorrência de sintomas osteomusculares nas variadas partes do corpo do indivíduo. Já para os escores de Dor a variação é de zero (sintoma sem nenhuma dor) a dez (sintoma com dor máxima)9.

Após a aplicação dos instrumentos, os dados foram tabulados em uma planilha com a utilização do software Excel (Microsoft 2013) e posteriormente analisados com o auxílio do pacote estatístico Statistical Package of Social Sciences (SPSS 24.0). A caracterização do perfil sociodemográfico eambiente organizacional foram realizadas por meio da frequência absoluta (n) erelativa (\%). A nãonormalidade dos dados foi verificada utilizando o teste de Shapiro-Wilk.

A comparação dos escores dos sintomas osteomusculares (Nórdico) com o perfil sociodemográfico e ambiente organizacional foi realizado utilizando os testes de Mann-Whitney ou KruskalWallis. No caso dos escores de Dor, 16 pessoas não referiram nenhum sintoma, logo eles não entraram nas análises comparativas. Em todas as análises foi adotado um nível de significância de 5\%.

A pesquisa foi registrada na Plataforma Brasil do Ministério da Saúde sob protocolo CAAE:62211016.5.0000.0037, aprovada pelo Comitê de Ética em Pesquisa da Pontifícia Universidade Católica de Goiás com o parecer no 1.875.691 e pelo Comitê de Ética em Pesquisa do Hospital Escola com o parecer n 1.906.747.

\section{Resultados}

Responderam ao presente estudo 119 indivíduos, sendo eles profissionais da equipe de enfermagem. A média de idade dos entrevistados foi de 35,9 anos $( \pm 9,2)$. A faixa etária predominante foi de 20 a 35 anos, caracterizando uma população relativamente jovem. Em relação ao núcleo familiar, $63 \%$ dos trabalhadores relataram ter filhos e sobre os hábitos de vida, 51,3\% disseram realizar algum tipo de atividade física regularmente (Tabela 1).

Tabela 1. Caracterização do perfil sociodemográfico dos 119 profissionais de enfermagem de um hospital escola. Goiânia, Goiás, Brasil, 2018.

\begin{tabular}{lcc}
\hline Variáveis & N & $\%$ \\
\hline Faixa etária & 63 & \\
\hline 20 a 35 anos & 56 & 52,9 \\
\hline 36 a 63 anos & 116 & 47,1 \\
\hline Gênero & 3 & 97,5 \\
\hline Feminino & & 2,5 \\
\hline Masculino & 7 & 5,9 \\
\hline Setor do hospital & & \\
\hline Administrativo & & \\
\hline
\end{tabular}




\begin{tabular}{|c|c|c|}
\hline Cirurgia & 13 & 10,9 \\
\hline Internação & 69 & 58,0 \\
\hline Urgência e Emergência & 30 & 25,2 \\
\hline \multicolumn{3}{|l|}{ Renda mensal } \\
\hline 1 a 3 salários & 111 & 93,3 \\
\hline 4 a 8 salários & 8 & 6,7 \\
\hline \multicolumn{3}{|l|}{ Estado civil } \\
\hline Casado & 45 & 37,8 \\
\hline Separado & 19 & 16,0 \\
\hline Solteiro & 55 & 46,2 \\
\hline \multicolumn{3}{|l|}{ Tem filhos } \\
\hline Não & 44 & 37,0 \\
\hline Sim & 75 & 63,0 \\
\hline \multicolumn{3}{|l|}{ Atividade física } \\
\hline Não & 58 & 48,7 \\
\hline Sim & 61 & 51,3 \\
\hline \multicolumn{3}{|l|}{ Tarefas domésticas } \\
\hline Não & 3 & 2,5 \\
\hline Sim & 116 & 97,5 \\
\hline
\end{tabular}

Em relação ao ambiente organizacional, foram entrevistados 25 enfermeiros e 94 técnicos de enfermagem. Um total de 45,4\% dos entrevistados relatou possuir de um a cinco anos de tempo de serviço, sendo essa porcentagem igual tanto para o tempo de serviço na enfermagem quanto para o tempo de serviço no hospital. Cerca de 71,4\% dos entrevistados relataram não possuírem outro emprego, $89,1 \%$ acreditaram que o trabalho executado trazia algum risco à saúde, porém 63\% disseram estar satisfeitos no trabalho e $89,9 \%$ consideraram o ambiente de trabalho como bom/excelente (Tabela 2).

Tabela 2. Caracterização do ambiente organizacional dos 119 profissionais de enfermagem de um hospital escola. Goiânia, Goiás, Brasil, 2018.

\begin{tabular}{|lcc|}
\hline Variáveis & N & $\%$ \\
\hline Contrato (hospital) & & \\
\hline Enfermeiro (nível superior) & 25 & 21,0 \\
Técnico em enfermagem & 94 & 79,0 \\
\hline
\end{tabular}

Tempo de serviço (enfermagem)

\begin{tabular}{lcc|} 
Menos de 1 ano & 18 & 15,1 \\
1 a 5 anos & 54 & 45,4 \\
6 a 10 anos & 22 & 18,5 \\
\hline Acima de 10 anos & 25 & 21,0 \\
\hline Tempo de serviço (hospital) & & \\
\hline Menos de 1 ano & 37 & 31,1 \\
\hline 1 a 5 anos & 54 & 45,4 \\
\hline 6 a 10 anos & 12 & 10,1 \\
\hline Acima de 10 anos & 16 & 13,4 \\
\hline Jornada de trabalho (horas) & & \\
\hline 40 horas & 7 & 5,9 \\
\hline 44 horas & 112 & 94,1 \\
\hline Outro emprego & & \\
\hline Não & 106 & 89,1 \\
\hline Sim & 85 & 71,4 \\
\hline Risco à saúde & 34 & 28,6 \\
\hline Não & & \\
\hline Sim & 13 & \\
\hline
\end{tabular}

Satisfação no trabalho

\begin{tabular}{lcc} 
Insatisfeito & 28 & 23,5 \\
Indiferente & 7 & 5,9 \\
Satisfeito & 75 & 63,0 \\
Muito satisfeito & 9 & 7,6 \\
\hline Ambiente de trabalho & & \\
\hline Péssimo & 1 & 0,8 \\
Ruim & 11 & 9,2 \\
Bom & 99 & 83,2 \\
Excelente & 8 & 6,7 \\
\hline Costuma se cansar & & \\
\hline Não & 40 & 33,6 \\
\hline Sim & 79 & 66,4 \\
\hline
\end{tabular}

No que concerne à prevalência de sintomas osteomusculares e média de dor, $16(13,4 \%)$ pessoas não apresentaram nenhum sintoma e $103(86,6 \%)$ pessoas referiram algum tipo de sintoma. Nos últimos 12 meses, 60,5\% relataram dor na região lombar, $54,6 \%$ no pescoço e $46,2 \%$ nos ombros. Nos últimos sete dias, $43,7 \%$ referiram dor lombar, $28,6 \%$ no tor- 
nozelo/pés e $26,9 \%$ nos ombros, sendo a dor na região lombar a maior causa de afastamento do trabalho (26,9\%) (Tabela 3 ).

Tabela 3. Prevalência de sintomas osteomusculares e média de dor dos profissionais de enfermagem de um hospital escola. Goiânia, Goiás, Brasil, 2018.

\begin{tabular}{lcccc}
\hline & 12 meses & 7 dias & Afastamento & Dor* \\
Variáveis & $\mathrm{n}(\%)$ & $\mathrm{n}(\%)$ & $\mathrm{n}(\%)$ & (Média $\pm \mathrm{DP})$ \\
Pescoço & $65(54,6)$ & $21(17,6)$ & $16(13,4)$ & $4,71 \pm 2,31$ \\
Ombros & $55(46,2)$ & $32(26,9)$ & $18(15,1)$ & $5,25 \pm 2,51$ \\
Cotovelo & $10(8,4)$ & $6(5,0)$ & $2(1,7)$ & $6,36 \pm 2,54$ \\
Punho/Mãos & $42(35,3)$ & $25(21,0)$ & $14(11,8)$ & $4,77 \pm 2,21$ \\
Torácica & $24(20,2)$ & $17(14,3)$ & $9(7,6)$ & $5,03 \pm 2,46$ \\
Lombar & $72(60,5)$ & $52(43,7)$ & $32(26,9)$ & $5,54 \pm 2,65$ \\
Ancas/Coxas & $33(27,7)$ & $18(15,1)$ & $13(10,9)$ & $5,40 \pm 2,68$ \\
Joelhos & $39(32,8)$ & $23(19,3)$ & $21(17,6)$ & $5,80 \pm 2,82$ \\
Tornozelo/Pés & $47(39,5)$ & $34(28,6)$ & $18(15,1)$ & $5,98 \pm 2,78$ \\
\hline
\end{tabular}

* Respostas negativas foram omitidas nas frequências.

Os dados desta pesquisa revelaram uma amostra homogênea da população estudada e mostraram por meio da comparação entre os escores dos sintomas osteomusculares com o ambiente organizacional, que os trabalhadores que relataram algum sintoma nos últimos 12 meses também consideraram que o seu trabalho traz algum risco à saúde $(p=0,02)$. Além disso, os entrevistados que informaram que "costumam se cansar no ambiente de trabalho" também apresentaram algum sintoma osteomuscular nos últimos 12 meses $(p<0,01)$ e nos últimos sete dias $(p=0,02)$. Em relação à satisfação no ambiente de trabalho, a pesquisa identificou que os profissionais que afirmaram estarem satisfeitos ou muito satisfeitos apresentaram menos sintomas osteomusculares nos últimos sete dias $(p=0,05)$ e tendência nos últimos 12 meses $(p=0,06)$ (Tabela 4 ).

Tabela 4. Resultados da comparação dos escores dos sintomas osteomusculares com o ambiente organizacional dos profissionais de enfermagem de um hospital escola. Goiânia, Goiás, Brasil, 2018.
Nórdico (Média \pm Desvio padrão)

\begin{tabular}{|c|c|c|c|c|}
\hline \multirow[t]{2}{*}{$\begin{array}{l}\text { Ambiente } \\
\text { organizacional }\end{array}$} & \multirow{2}{*}{$\begin{array}{l}12 \text { meses } \\
(\mathrm{n}=119)\end{array}$} & \multirow{2}{*}{$\begin{array}{l}7 \text { dias } \\
(n=119)\end{array}$} & \multirow{2}{*}{$\begin{array}{l}\text { Afastamento } \\
\qquad(\mathrm{n}=119)\end{array}$} & \multirow{2}{*}{$\begin{array}{c}\text { Dor } \\
(n=103)\end{array}$} \\
\hline & & & & \\
\hline \multicolumn{5}{|c|}{ Contrato de trabalho* } \\
\hline $\begin{array}{l}\text { Enfermeiro } \\
\text { (nível superior) }\end{array}$ & $13,08 \pm 2,27$ & $11,44 \pm 2,24$ & $9,84 \pm 1,37$ & $4,83 \pm 1,89$ \\
\hline $\begin{array}{l}\text { Técnico em } \\
\text { enfermagem }\end{array}$ & $13,13 \pm 3,27$ & $11,35 \pm 2,91$ & $10,49 \pm 2,24$ & $5,09 \pm 2,06$ \\
\hline pvalor & 0,84 & 0,50 & 0,26 & 0,72 \\
\hline \multicolumn{5}{|c|}{ Tempo de serviço (enf)** } \\
\hline Menos de 1 ano & $12,06 \pm 2,65$ & $10,56 \pm 1,58$ & $9,50 \pm 0,86$ & $3,65 \pm 1,18$ \\
\hline 1 a 5 anos & $13,70 \pm 2,96$ & $11,61 \pm 2,96$ & $10,61 \pm 2,19$ & $5,19 \pm 1,96$ \\
\hline 6 a 10 anos & $13,05 \pm 3,50$ & $11,64 \pm 3,16$ & $10,64 \pm 2,63$ & $5,33 \pm 2,06$ \\
\hline Acima de 10 anos & $12,68 \pm 3,12$ & $11,20 \pm 2,68$ & $10,16 \pm 1,91$ & $5,31 \pm 2,28$ \\
\hline pvalor & 0,33 & 0,87 & 0,52 & 0,97 \\
\hline \multicolumn{5}{|c|}{ Tempo de serviço (hospital)** } \\
\hline Menos de 1 ano & $12,62 \pm 3,01$ & $10,68 \pm 1,87$ & $10,05 \pm 1,78$ & $5,49 \pm 2,59$ \\
\hline 1 a 5 anos & $13,67 \pm 3,06$ & $12,00 \pm 3,13$ & $10,83 \pm 2,52$ & $5,10 \pm 2,01$ \\
\hline 6 a 10 anos & $12,83 \pm 3,35$ & $11,42 \pm 3,09$ & $9,92 \pm 1,44$ & $5,10 \pm 1,77$ \\
\hline Acima de 10 anos & $12,63 \pm 3,07$ & $10,81 \pm 2,74$ & $9,75 \pm 1,24$ & $4,66 \pm 1,81$ \\
\hline$p$ valor & 0,39 & 0,18 & 0,36 & 0,56 \\
\hline \multicolumn{5}{|c|}{ Jornada de trabalho (horas) ${ }^{* *}$} \\
\hline 40 horas & $13,57 \pm 3,15$ & $12,14 \pm 3,29$ & $9,57 \pm 0,98$ & $4,83 \pm 2,66$ \\
\hline 44 horas & $13,09 \pm 3,09$ & $11,32 \pm 2,75$ & $10,40 \pm 2,14$ & $5,04 \pm 1,98$ \\
\hline pvalor & 0,68 & 0,60 & 0,34 & 0,92 \\
\hline \multicolumn{5}{|l|}{ Outro emprego* } \\
\hline Não & $12,91 \pm 2,95$ & $11,38 \pm 2,69$ & $10,24 \pm 1,96$ & $4,98 \pm 1,98$ \\
\hline Sim & $13,65 \pm 3,37$ & $11,35 \pm 3,01$ & $10,65 \pm 2,42$ & $5,13 \pm 2,10$ \\
\hline pvalor & 0,33 & 0,71 & 0,41 & 0,76 \\
\hline \multicolumn{5}{|l|}{ Risco à saúde* } \\
\hline Não & $11,23 \pm 2,09$ & $10,15 \pm 1,46$ & $10,31 \pm 1,44$ & $4,55 \pm 2,08$ \\
\hline Sim & $13,35 \pm 3,11$ & $11,52 \pm 2,86$ & $10,36 \pm 2,17$ & $5,08 \pm 2,01$ \\
\hline pvalor & 0,02 & 0,11 & 0,55 & 0,39 \\
\hline \multicolumn{5}{|c|}{ Satisfação no trabalho** } \\
\hline Insatisfeito & $13,86 \pm 3,21$ & $12,00 \pm 2,97$ & $10,71 \pm 2,75$ & $5,18 \pm 1,90$ \\
\hline Indiferente & $15,29 \pm 2,63$ & $13,71 \pm 3,99$ & $10,29 \pm 1,80$ & $5,10 \pm 1,91$ \\
\hline Satisfeito & $12,76 \pm 2,99$ & $10,96 \pm 2,40$ & $10,23 \pm 1,92$ & $4,63 \pm 2,56$ \\
\hline Muito satisfeito & $12,11 \pm 2,98$ & $11,00 \pm 3,20$ & $10,33 \pm 1,50$ & $5,03 \pm 2,05$ \\
\hline$p$ valor & 0,06 & 0,05 & 0,94 & 0,91 \\
\hline
\end{tabular}




\begin{tabular}{lrrrr}
\multicolumn{1}{l}{ Ambiente de trabalho** } & & & \\
Ruim & $14,27 \pm 2,94$ & $11,45 \pm 3,45$ & $12,00 \pm 3,29$ & $5,592,42$ \\
Bom & $13,06 \pm 3,12$ & $11,32 \pm 2,72$ & $10,21 \pm 1,90$ & $4,971,94$ \\
Excelente & $11,75 \pm 2,19$ & $11,13 \pm 2,03$ & $10,00 \pm 1,77$ & $4,151,61$ \\
p valor & 0,23 & 0,28 & 0,23 & 0,36 \\
Costuma se cansar* & & & & \\
\hline Não & $11,88 \pm 2,89$ & $10,63 \pm 2,38$ & $9,75 \pm 1,33$ & $4,561,95$ \\
Sim & $13,75 \pm 2,99$ & $11,75 \pm 2,89$ & $10,66 \pm 2,34$ & $5,232,02$ \\
\hline v valor & $<0,01$ & 0,02 & 0,06 & 0,11 \\
\hline
\end{tabular}

*Mann-Whitney;

**Kruskal-Wallis

\section{Discussão}

Por estarem relacionado intrinsicamente à função exercida, ao ambiente físico e emocional de trabalho e às condições oferecidas aos trabalhadores, as LER/DORT varia de prevalência significativamente em diferentes profissões. Neste sentido, os enfermeiros e técnicos de enfermagem são grupo de profissionais que são considerados alvo fácil para desenvolvimento de tais condições, uma vez que estão submetidos a longas jornadas de trabalho, prolongada exposição a estressores físicos e psicológicos, realizam movimentos repetitivos, adotam posturas inadequadas e trabalham muitas vezes em períodos noturnos, que deveriam ser reservados para descanso ${ }^{4}$.

Segundo uma pesquisa realizada pela Fundação Oswaldo Cruz (FIOCRUZ) e o Conselho Federal de Enfermagem (COFEN) em 2015, existia na época 1,6 milhão de profissionais de enfermagem no Brasil na época e destes, 20\% (320 mil) eram compostos por enfermeiros e o demais por técnicos e auxiliares. No nosso estudo, verificamos uma porcentagem semelhante: $21 \%$ da equipe era composta de enfermeiros e o restante por técnicos. Além disso, a maioria $(84,6 \%)$ dos profissionais de enfermagem no Brasil pertence ao gênero feminino, o que também vai de encontro com o predomínio feminino nos nossos entrevistados ${ }^{11}$.

Este predomínio do sexo feminino não é apenas um dado histórico da profissão, como também é um dos fatores que podem contribuir para sintomas osteomusculares mais exuberantes. Isto porque a mulher geralmente é responsável pelo trabalho doméstico, configurando-se o que se chama de jornada dupla de trabalho. Esta desigualdade na relação de trabalho acaba favorecendo o desenvolvimento de agravos e comorbidades ${ }^{12}$. Os resultados do presente estudo endossam essa perspectiva, na medida que as mulheres que possuíam filhos se afastaram mais do trabalho do que aquelas que não possuíam.

De modo geral, os papeis sociais atribuídos a homens e mulheres no ambiente domiciliar, os salários mais baixos aliados ao baixo reconhecimento social, além de fatores biológicos influenciam diretamente no aparecimento dos sintomas osteomusculares relacionados ao trabalho. Um estudo de âmbito nacional mostrou maior prevalência de DORT em mulheres e, dentre elas as que viviam sem companheiro, apresentaram menor ocorrência de sintomas. Concluiu-se, então, que as tarefas domésticas seriam mais prováveis na vida em casal, por vezes com filhos podendo justificar mais sintomas osteomusculares ${ }^{13}$.

Quanto ao local do corpo mais acometido por sintomas osteomusculares nos últimos 12 meses, a lombar, o pescoço e os ombros foram as áreas mais acometidas correspondendo a $60,5 \%, 54,6 \%$ e $46,2 \%$ respectivamente. Em um estudo na China com quase 7000 enfermeiros e técnicos de enfermagem, estas três áreas foram também as mais afetadas, correspondendo por $62,71 \%, 59,77 \%$ e $49,66 \%$ dos sintomas osteomusculares no ano anterior. Segundo o autor, esta frequência está relacionada a atividade dos profissionais de enfermagem de carregar pacientes e transferi-los de uma maca a outra, o que acaba gerando mecanismos de compensação e sobrecarregando alguns grupos musculares ${ }^{7}$.

Uma pesquisa buscou comparar medidas de intervenção com a diminuição das queixas álgicas em profissionais de enfermagem. Foram selecionadas enfermeiras de um hospital em Tel Aviv e as separaram em dois grupos: um grupo que recebeu intervenções ergonômicas personalizadas com alguns exercícios físicos e um grupo controle. As participantes foram fotografadas fazendo as tarefas diárias antes e depois da intervenção. Neste estudo, a intervenção mostrou ser efetiva em reduzir os fatores de risco para as desordens musculoesqueléticas, mas não houve diferença significativa no número de grupos corporais com dor ${ }^{14}$.

Em outro estudo foi ampliada a complexidade da intervenção e envolveram, além do treinamento fisioterápico, acompanhamento psicossocial, com o intuito de avaliar o impacto na prevenção de dores osteomusculares. Para tanto, dividiu as enfermeiras em um grupo que só receberia treinamento fisioterápico e em outro grupo que além da fisioterapia teria também sessões de psicoterapia. Como resultado, o grupo de intervenção mostrou uma melhora significativa na intensidade da dor dos movimentos cotidianos quando comparado com o grupo controle e as melhorias aumentaram ainda mais nas 12 semanas após a intervenção ${ }^{15}$.

Além de intervenções psicossociais, pausas suplementares (30 segundos a 5 minutos) mostraram ser mais efetivas que $o$ cronograma de folga que alguns trabalhos convencionalmente determinam. Estas pausas não impactam negativamente a produção e são efetivas em diferentes conformações laborais ${ }^{16}$. 
Outro resultado que merece destaque no presente estudo foi que a maioria dos trabalhadores que tiveram algum sintoma consideraram que o seu trabalho traz algum risco à saúde. Esta percepção sobre o ambiente laboral e sua influência na assistência de enfermagem está presente desde o século XIX quando Florence Nightingale criou a Teoria Ambientalista. Segundo ela, um ambiente adequado era o diferencial na recuperação dos doentes, uma vez que todas as condições e influências externas do local de trabalho são capazes de melhorar o cuidado dos enfermeiros com os doentes e prevenir ou contribuir para a doença e a morte ${ }^{17}$.

Além disso, os profissionais de enfermagem representam a maior força de trabalho de uma instituição hospitalar e para tanto precisam trabalhar em um ambiente que os assegurem proteção. Urge, portanto, investigar e eliminar os riscos à saúde do ambiente laboral destes profissionais e proporcionar condições mais confortáveis. Os benefícios não se restringiriam a uma melhor qualidade de vida por parte dos trabalhadores, melhores desfechos aos pacientes, mas também uma redução nos custos devido à licença-saúde, readaptações e até mesmo aposentadoria por invalidez ${ }^{12}$.

A análise dos resultados mostrou ainda que os profissionais que afirmaram estarem satisfeitos ou muito satisfeitos apresentaram menos sintomas osteomusculares nos últimos sete dias e tendência nos últimos 12 meses. Esta associação reflete a grande influência do componente emocional nos sintomas físicos já citado nesta discussão e comprovado com o resultado deste estudo. Uma pesquisa comprovou por meio de análise de regressão logística múltipla que estresse e ansiedade aumentavam o risco de doenças osteomusculares relacionadas a trabalho em até duas vezes ${ }^{18}$. Além disso, outro estudo realizado na China mostrou que boa condição psicológica é fator protetor para as DORTs, juntamente com boa condição de saúde, quantidade adequada de trabalhadores, suporte financeiro e social ${ }^{19}$.

Enquanto a satisfação no trabalho atua como mecanismo protetor, a análise do nosso estudo mostrou que os entrevistados que informaram que "costumam se cansar no ambiente de trabalho" apresentaram algum sintoma osteomuscular nos últimos 12 meses e nos últimos sete dias. Esse dado mostra a relação da fadiga auto-referida com os sintomas físicos experenciados pelos entrevistados. A fadiga é, portanto, não apenas uma consequência do trabalho excessivo, mas também uma causa de sintomas osteomusculares relacionados ao trabalho que atinge não só o sistema muscular, como também pode estar relacionado a insônia e dor crônica, diminuindo assim, a qualidade de vida dos trabalhadores ${ }^{20}$.

\section{Conclusão}

Analisando os dados sobre os sintomas osteomusculares em profissionais de enfermagem em um hospital escola, este estudo mostrou ser o gênero feminino o gênero dominante entre os profissionais, assim como a formação técnica mais comum do que a formação de nível superior em enfermagem. A maioria dos trabalhadores executavam uma jornada de 44 horas semanais, não possuíam outro emprego e se diziam satisfeito ou muito satisfeitos.

Foi notado uma alta prevalência de algum tipo de sintoma osteomuscular e as regiões corporais mais atingidas nos últimos 12 meses foram a lombar, o pescoço e os ombros. Já nos últimos sete dias as regiões mais acometidas foram a lombar, o tornozelo/pés e os ombros. A percepção de risco à saúde no ambiente de trabalho e sentimento de cansaço ou fadiga foram os fatores implicados em mais sintomas, enquanto que a satisfação no trabalho esteve relacionada a menos queixas álgicas.

Nota-se, portanto, que urge dar mais atenção ao ambiente laboral no qual os enfermeiros estão inseridos e em medidas que diminuiriam o impacto do trabalho na qualidade de vida destes profissionais, refletida neste estudo, em dores osteomusculares. Faz se necessário compreender que os profissionais de enfermagem estão submetidos a constantes agente estressores físico e emocionais e que sua saúde possui impacto direto na saúde de seus pacientes.

Afinal, cuidar de quem cuida é tão necessário e importante quanto cuidar dos enfermos. Uma boa saúde humanizada envolve também o preparo de um bom ambiente hospitalar, que possibilite uma boa convivência entre os profissionais, bem como um ambiente que não imponha risco à saúde $e$ que previna doenças relacionadas ao trabalho.

\section{Agradecimentos}

À Fundação de Amparo à Pesquisa do Estado de Goiás (FAPEG) concedeu Bolsa de Mestrado. A Pontifícia Universidade Católica de Goiás (PUC Goiás) a Bolsa de Iniciação Científica.

\section{Referências Bibliográficas}

1.Instituto Nacional do Seguro Social. Instrução Normativa INSS/DC n. 98 de 5 de dezembro de 2003. Aprova norma técnica sobre lesões por esforços repetitivos-LER ou distúrbios osteomusculares relacionados ao trabalho- DORT. Diário Oficial da República Federativa do Brasil, Brasília; 2003.

2. Brasil. Pesquisa Nacional de Saúde, 2013: percepção do estado de saúde, estilos de vida e doenças crônicas. Brasília: Ministério da Saúde; 2013.

3. Brasil. Primeiro boletim quadrimestral sobre benefícios por incapacidade: dia mundial em memória às vítimas de acidentes 
de trabalho. Brasília: Ministério da Previdência Social; 2014. 4. Ribeiro T, Serranheira F, Loureiro H. Work related musculoskeletal disorder in primary health care nurses. Appl Nurs Res. 2017;33:72-7.

5. Thinkhamrop W, Sawaengdee K, Tangcharoensathien V, Theerawit T, Laohasiriwong W, Saengsuwan J, et al. Burden of musculoskeletal disorders among registered nurses: evidence from the Thai nurse cohort study. BMC Nurs. 2017;16(68):1-9. 6. Rahimi A, Vazini H, Alhani F, Anoosheh M. Relationship between low back pain with quality of life, depression, anxiety and stress among emergency medical technicians. Trauma Mon. 2015;20(2):27-32.

7. Yan P, Li F, Zhang L, Yang Y, Huang A, Wang Y, et al. Prevalence of work-related musculoskeletal disorders in the nurses working in hospital of Xinjiang Uygur autonomous region. Pain Res Manag. 2017;1-7.

8. Amin NA, Rusli BN, Noah RM, Oxley JA, Quek KF. Work related musculoskeletal disorders in female nursing personnel: prevalence and impact. Int J Collab Res Internal Med Public Health. 2016;8(3):294-315.

9. Pinheiro FA, Troccoli BT, Carvalho CV. Validação do Questionário Nórdico de Sintomas Osteomusculares como medida de morbidade. Rev. Saúde Pública. 2002;36(3):2017-12.

10. Barros ENC, Alexandre NMC. Cross-cultural adaptation of the nordic musculoskeletal questionnaire. Int Nurs Rev. 2003;50(2):101-8.

11. Fundação Oswaldo Cruz, Conselho Federal de Enfermagem. Pesquisa perfil da enfermagem no Brasil. 2015. Disponível em: <http://www.cofen.gov.br/perfilenfermag em/>. Acesso em: 10 jul. 2018.

12. Pereira AB, Karino ME, Martins JT, Scholze AR, Galdino MJQ, Ribeiro RP. Perfil de trabalhadores readaptados em um hospital público do Sul do Brasil. Rev Bras Med Trab. 2017;15(4):317-23. 13. Assunção $A A$, Abreu MNS. Fatores associados a distúrbios osteomusculares relacionados ao trabalho autorreferidos em adultos brasileiros. Rev Saude Pública. 2017;51(supl. 1:10s):1-12. 14. Ratzon NZ, Bar-Niv NA, Froom P. The effect of a structure personalized ergonomic intervention program for hospital nurses with reported musculoskeletal pain: as assigned randomized control trial. Work. 2016;54(2):367-77.

15. Becker A, Angerer P, Muller A. The prevention of musculoskeletal complaints: a randomized controlled trial on additional effects of a work-related psychosocial coaching intervention compared to physiotherapy alone. Int Arch Occup Environ Health. 2017;90(4):357-71.

16. Stock SR, Nicolakakis N, Vézina N, Vézina M, Gilbert L, Turcot $A$, et al. Are work organization interventions effective in preventing or reducing work-related musculoskeletal disorders?
A systematic review of the literature. Scand J Work Environ Health. 2018;44(2):113-33.

17. Medeiros ABA, Enders BC, Lira ALBC. Teoria ambientalista de Florence Nightingale: uma análise crítica. Esc Anna Nery. 2015;19(3):518-24.

18. Amin NA, Quek KF, Oxley JA, Noah RM, Nordin R. Emotional distress as a predictor of work-related musculoskeletal disorders in Malaysian nursing professionals. Int J Occup Environ Med. 2018;9(2):69-78.

19. Yan P, Yang Y, Zhang L, Li F, Huang A, Wang Y, et al. Correlation analysis between work-related musculoskeletal disorder and the nursing practice environment, quality of life, and social support in the nursing professional. Medicine (Baltimore). 2018;97(9):e0026.

20. Skarpsno ES, Nilsen TIL, Sand T, Hagen K, Mork PJ. Physical work exposure, chronic musculoskeletal pain and risk of insomnia: longitudinal data from the HUNT study, Norway. Occup Environ Med. 2018;75(6):421-6. 